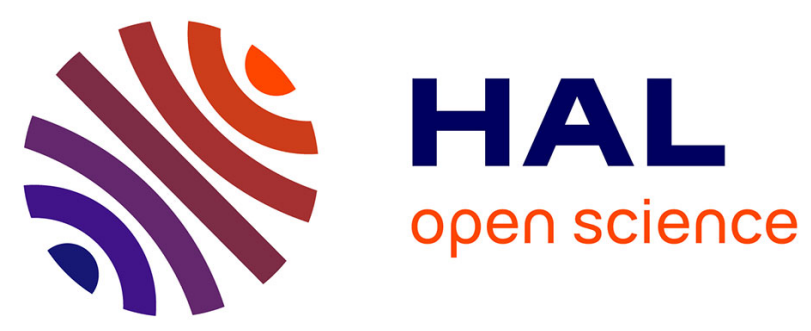

\title{
Nos performances de conduite sont-elles sous l'influence du tempo de la musique que nous écoutons ? Une étude sur simulateur
}

Jordan Navarro, Mathieu Lesourd, François Osiurak, Emanuelle Reynaud

\section{- To cite this version:}

Jordan Navarro, Mathieu Lesourd, François Osiurak, Emanuelle Reynaud. Nos performances de conduite sont-elles sous l'influence du tempo de la musique que nous écoutons ? Une étude sur simulateur. RTS - Recherche Transports Sécurité, 2016, Distractions au volant, 2015 (03-04), pp.211221. 10.4074/S0761898016004076 . hal-01670600

\section{HAL Id: hal-01670600 https://hal.science/hal-01670600}

Submitted on 21 Dec 2017

HAL is a multi-disciplinary open access archive for the deposit and dissemination of scientific research documents, whether they are published or not. The documents may come from teaching and research institutions in France or abroad, or from public or private research centers.
L'archive ouverte pluridisciplinaire HAL, est destinée au dépôt et à la diffusion de documents scientifiques de niveau recherche, publiés ou non, émanant des établissements d'enseignement et de recherche français ou étrangers, des laboratoires publics ou privés. 


\title{
Nos performances de conduite sont-elles sous l'influence du tempo de la musique que nous écoutons? Une étude sur simulateur
}

\section{Are driving performances under musical tempo influence? A simulator study}

\author{
Jordan Navarro · Mathieu Lesourd · François Osiurak · Emanuelle Reynaud \\ (C) IFSTTAR et Éditions NecPlus 2015
}

Résumé La musique accompagne fréquemment nos déplacements automobiles, mais influence-t-elle nos performances de conduite? En fonction du contexte, la musique peut être envisagée comme bénéfique (en nous stimulant lorsque nécessaire par exemple) ou au contraire pénalisante (en nous gênant lors d'une manœuvre de stationnement difficile). Compte tenu de la complexité du stimulus musical et de ses multiples effets sur nos comportements, une compréhension globale des effets de la musique sur les comportements de conduite demande d'abord de considérer les différentes dimensions de la musique une à une sur les différentes tâches de conduite. Dans le cadre de cette étude, nous nous sommes focalisés sur les effets du tempo seul lors d'une tâche de suivi d'un véhicule. Le tempo d'un morceau musical choisi par les participants a ainsi été modifié dans l'hypothèse qu'une accélération de tempo serait accompagnée d'une augmentation d'activation (arousal) et inversement pour une décélération de tempo. Le niveau d'activation étant lié aux performances, le comportement de suivi d'un véhicule s'en trouverait donc affecté. Les résultats indiquent que la musique jouée en situation de conduite simulée et son tempo sont en mesure de modifier le niveau d'activation des conducteurs. Toutefois les performances de

Jordan Navarro $(\bowtie)$

Université de Lyon, Laboratoire d'étude des mécanismes cognitifs

5 avenue Pierre Mendès France, 69676 Bron Cedex, France

e-mail : jordan.navarro@univ-lyon2.fr

Mathieu Lesourd $(\bowtie)$

Université de Lyon, Laboratoire d'étude des mécanismes cognitifs

5 avenue Pierre Mendès France, 69676 Bron Cedex, France

e-mail : mathieu.lesourd@univ-lyon2.fr

François Osiurak $(\bowtie)$

Institut Universitaire de France

103 Boulevard Saint-Michel, 75005 Paris, France

e-mail : francois.osiurak@univ-lyon2.fr

Emanuelle Reynaud $(\bowtie)$

Université de Lyon, Laboratoire d'étude des mécanismes cognitifs

5 avenue Pierre Mendès France, 69676 Bron Cedex, France

e-mail : emanuelle.reynaud@univ-lyon2.fr conduite ne semblent pas affectées par les modifications d'activation générées par la musique. Dans le futur, il serait intéressant d'investiguer les effets du tempo musical dans d'autres situations de conduite.

Mots clés Musique · tempo · tâche de suivi · guidage · conduite automobile

Abstract Is the music we are often listening to while driving influences our driving performances? Depending on the context, music can be seen as helping (e.g., by arousing a driver that need so) or harming (e.g., by interfering with a parking manoeuvre) driving performance. Because music is a very complex stimulus with many effects on our behaviour, its effects on driving cannot be understood at the first glance. A decomposition of the various music dimensions on the different driving tasks is required. In this study, the focus was placed on music tempo in a car following task. We conducted a study where the tempo of a musical track, chosen by participants, was modified (increased and decreased). It was hypothesised that an increased tempo would lead to an increased arousal and the reverse for a decreased tempo. Because arousal is related to performance, car following performances should be impacted by arousal changes. Results showed that music played while driving in a car simulator and its tempo is impacting drivers' arousal. However driving performance was not affected by these arousal changes. Future studies should focus on the impact of music tempo in different driving contexts.

Keywords Music $\cdot$ Tempo $\cdot$ Following task $\cdot$ Steering control · Car driving

\section{Introduction}

Jouer et écouter de la musique est une activité culturelle majeure quelles que soient les civilisations et les périodes de l'histoire considérées [1]. Dans nos sociétés modernes, l'enceinte d'un véhicule automobile personnel 
ou professionnel est un lieu d'écoute musical privilégié qui participe au sentiment d'être chez soi dans son véhicule [2]. L'habitacle d'un véhicule automobile est même le premier lieu d'écoute musical déclaré avant l'écoute à la maison, pendant l'exercice physique ou avec des amis [3]. Ainsi près de $75 \%$ des conducteurs écoutent de la musique ou la radio lorsqu'ils sont au volant [4].

La musique est un objet d'étude en soi. Il n'est pas question ici de chercher à comprendre la complexité, la richesse ni même l'ensemble des effets de la musique sur notre cognition et nos comportements. Nous considérerons uniquement la musique comme un stimulus qui se surimpose à l'activité qui nous intéresse : la conduite automobile. Il est alors question de la musique comme une activité "d'arrière-plan » ou background music. L'écoute musicale est souvent combinée avec une autre activité comme déjeuner au restaurant, faire du shopping, ou encore faire de l'exercice physique. Plusieurs de ces situations ont fait l'objet d'études visant à mieux comprendre l'influence de la musique sur une autre activité. Pour la restauration par exemple, il a été montré une augmentation du nombre de bouchées avec l'augmentation du tempo de la musique sans même que les individus déclarent être conscients de la musique jouée, ou encore une modification de la durée du repas en fonction de l'adéquation des préférences musicales à musique diffusée $[5,6]$. Les effets de la musique sur les comportements d'achat intéressent également les vendeurs [7]. Il a par exemple été observé une tendance à acheter plus de vin d'origine française ou allemande lorsqu'une musique typique du même pays était jouée dans l'enceinte du magasin de vin [8]. Concernant l'exercice physique, une musique rapide jouée à un volume élevé modifie la performance, au travers une augmentation de la vitesse de course et la fréquence cardiaque par exemple [9]. D'autres vertus sont également attribuées à la musique, qui peut aussi être utilisée à des fins thérapeutiques. Ce champ d'études, la musicothérapie, a notamment montré son intérêt dans le traitement de la démence $[10,11]$.

Compte tenu de la fréquence et de l'importance des déplacements automobiles dans la vie de la plupart des individus des sociétés modernes, les interactions entre écoute musicale et conduite automobile ont fait l'objet d'études variées. Plusieurs auteurs ont ainsi cherché à mesurer l'impact de la musique sur les performances de conduite. L'écoute musicale s'est avérée être positive ou neutre dans certains cas et négative dans d'autres.

D'un côté, il a été rapporté que l'écoute musicale en situation de conduite pouvait être bénéfique pour les tâches demandant une attention soutenue telles que le suivi d'un véhicule $[12,13]$. D'autres études concluent à un effet neutre de la musique sur le positionnement latéral dans la voie, la vitesse, les réponses à des événements dangereux, le temps mis pour freiner à l'apparition d'un signal lumineux
[14, 15]. D'un autre côté, des dégradations dans les performances de conduite ont été enregistrées lorsque les conducteurs étaient exposés à une écoute musicale à un volume élevé ou écoutaient leur musique préférée [16, 17]. L'écoute musicale peut donc avoir des effets très différents sur les performances de conduite. Aussi ces effets ne peuvent pas être considérés de manière globale car ils sont dépendants de la musique considérée, de l'activité de conduite et de leur éventuelle interaction.

Afin de mieux comprendre l'influence de la musique, qui est un stimulus multidimensionnel complexe, sur les performances de conduite, il semble donc nécessaire de considérer les différentes dimensions musicales isolément pour une activité de conduite donnée. La musique peut en effet être décrite selon plusieurs dimensions qui interagissent entre elles. Les principales propriétés d'une piste musicale en mesure d'influencer une autre activité sont : le tempo, le rythme, le mode, la hauteur, l'harmonie, le volume auquel la musique est jouée, et la valence émotionnelle qu'elle peut porter. Ces différents paramètres et leurs combinaisons sont en mesure d'affecter les comportements de conduite au travers plusieurs biais cognitifs. La musique est notamment connue pour être un inducteur émotionnel à même de dégrader les performances de conduite [18]. Les conducteurs se montrent également plus lents pour détecter des dangers lorsqu'un état émotionnel de colère a été induit via la musique [19]. Par ailleurs, les conducteurs se déplaçant dans un environnement complexe bénéficient plus de l'effet relaxant de morceaux musicaux apaisants lorsque ceux-ci sont introduits de manière abrupte que lorsqu'ils sont introduits progressivement [20].

Dans la présente étude, nous nous sommes focalisés sur une seule dimension de la musique jouée en fond sonore : le tempo. Le tempo est une composante essentielle de la musique et correspond à sa vitesse d'exécution. Il est exprimé en nombre de Battements par minute (BPM). Le BPM moyen d'une musique est classiquement compris entre : largo ou très lent et solennel (40 BPM ou moins) et prestissimo ou aussi vite que possible (200 BPM ou plus). Entre ces deux extrêmes les musiciens distinguent un éventail d'une quinzaine de nuances de tempo. Deux études ont déjà cherché à analyser l'effet du tempo de la musique sur les performances de conduite. Dans une première étude datant de la fin des années 1960, les performances de vingt-quatre conducteurs ont été comparées en situation de conduite réelle, sous trois conditions : sans musique, avec une musique lente et avec de la musique au tempo plus rapide (Tijuana Brass). L'activité de guidage, enregistrée au travers des actions sur le volant et sur les pédales de frein et d'accélérateur, a été modifiée en présence de la musique mais différemment en fonction du tempo de la musique considérée. Comparativement à la condition sans musique, une réduction des actions de guidage a été 
enregistrée avec les musiques lentes et une augmentation de ces mêmes actions a été rapportée avec une musique rapide. Par ailleurs, la vitesse de conduite moyenne augmentait quel que soit le tempo de la musique considéré [21]. Ces résultats ont mis en évidence un impact de la musique et de son tempo sur l'activité de conduite. Il s'agit d'une première pierre à l'édifice de la compréhension des effets de la musique sur les comportements de conduite. Pour mieux comprendre ces processus, une nouvelle étude réalisée dans un environnement urbain et périurbain simulé a été imaginée au début des années 2000. Dans cette étude quatre conditions sonores ont été réalisées (1) une condition contrôle sans musique (2) une condition avec des pistes musicales dites lentes avec des tempos allant de 40 à 70 BPM (3) une condition avec des pistes musicales aux tempos dits intermédiaires allant de 85 à 110 BPM (4) une condition avec des pistes musicales aux tempos dits rapides allant de 120 à 140 BPM [22]. Les pistes musicales ayant les tempos les plus rapides (120-140 BPM) ont entraîné les plus fortes augmentations de vitesse, de nombre de feux rouges non respectés et du nombre de franchissements de limites de voie de circulation en comparaison à la condition sans musique. La critique principale qui peut être formulée à l'encontre de cette étude réside dans le fait que la manipulation du tempo musical a été obtenue au travers de pistes musicales différentes. Ceci est généralement le cas lorsque le tempo musical est manipulé mais cela pose la question de savoir quelle part des résultats est attribuable au tempo musical seul et quelle part des résultats est attribuable à l'ensemble des autres paramètres qui caractérisent les différents morceaux musicaux utilisés dans les différentes conditions.

Afin de manipuler une seule dimension de la musique, le tempo, nous avons pris le parti dans la présente étude de modifier le tempo d'un même morceau musical. Les modifications de tempo effectuées ont été maintenues dans des proportions qui ne déforment pas la musique de manière trop importante, et ce afin de vérifier si le tempo est bien seul à l'origine des modifications des performances de conduites, comme enregistré dans les deux études présentées précédemment [21], [22]. Cette étude a donc été pensée pour évaluer l'impact du tempo musical sur l'activité de guidage du véhicule en faisant usage du même morceau musical joué à des tempos différents : pour cela, elle comporte donc trois conditions expérimentales musicales associées à trois versions du même morceau, différant uniquement par leurs tempos respectifs.

Si l'ensemble des rouages des effets de la musique sur nos activités cognitives et nos comportements ne sont pas connus, il semble que le tempo de la musique peut agir sur le niveau d'activation (c.-à-d. arousal en anglais) des individus. Du point de vue des processus attentionnels, le stimulus musical en situation de conduite automobile peut être envisagé de deux manières strictement opposées mais compatibles. La musique jouée en arrière-plan lors de la conduite peut agir à la fois comme un stimulant et comme un apaisant. Ces deux effets antagonistes de la musique en situation de conduite peuvent d'ailleurs être remarqués au quotidien par tous les conducteurs. En effet, ne vous est-il jamais arrivé de choisir une musique «stimulante » lors d'un trajet de nuit afin d'essayer de maintenir un bon niveau d'activation ? À l'inverse vous est-il déjà arrivé de couper l'autoradio lorsque vous cherchiez un endroit inconnu en ville par exemple? Au travers de ces deux exemples auxquels la plupart des conducteurs ont déjà certainement été confrontés, il apparaît clairement que les effets de la musique peuvent être distincts en fonction de la situation considérée.

Une grille de lecture cognitive de ces effets non uniformes de la musique sur la conduite réside dans l'utilisation des ressources attentionnelles dont dispose le conducteur et particulièrement le niveau d'activation inspiré de la loi dite en «U inversé » de Yerkes et Dodson [23]. Si un conducteur n'est que faiblement activé, il est attendu que la musique améliore ses capacités attentionnelles. À l'inverse si un conducteur est déjà fortement activé, la musique agira à la manière d'une tâche ajoutée et viendra diminuer les capacités attentionnelles du conducteur. Le tempo est connu pour être la composante musicale ayant le plus fort impact sur les comportements humains [24]. Un tempo musical lent est à même de diminuer le niveau d'arousal alors qu'un tempo musical rapide permet d'augmenter le niveau d'activation [25, 27]. En suivant la théorie proposée par Kahneman [28], le niveau d'activation agit comme un modulateur de la quantité de ressources attentionnelles qui peuvent être allouées à une ou plusieurs tâches. Plus un individu a un niveau d'activation important, plus la quantité de ressources attentionnelles disponibles augmente. La modulation du niveau d'activation générée par le tempo de la musique devrait donc se répercuter sur la quantité de ressources attentionnelles disponibles chez les individus. Ainsi une décélération du tempo devrait entraîner une diminution du niveau d'activation et donc de la quantité de ressources attentionnelles disponibles pour la tâche. À l'inverse, une accélération du tempo devrait entraîner une augmentation du niveau d'activation et donc de la quantité de ressources attentionnelles disponibles pour la tâche. Cependant, comme les ressources attentionnelles sont limitées leur augmentation est donc plafonnée [28]. De plus un niveau d'activation très élevé entraîne également une dégradation des performances [23, 29]. Même si une partie des mécanismes cognitifs à l'œuvre restent à expliquer, la diminution et l'augmentation des ressources attentionnelles disponibles peuvent toutes 
deux entraîner des dégradations de la performance via une sous-activation ou une sur-activation respectivement [29].

Par ailleurs, plusieurs études ont montré l'existence d'un lien entre le niveau d'activation et les préférences musicales [30]. Il a notamment été montré que la musique préférée augmente le niveau d'activation [31] et que plusieurs variables physiologiques, dont la fréquence cardiaque, corrèlent positivement avec la préférence pour la musique [32]. De plus la préférence musicale des individus va très largement à des musiques joyeuses qui sont habituellement associées à des tempos rapides, le tempo étant lui-même associé à une augmentation du niveau d'activation [25, 33]. Ainsi l'utilisation d'une piste musicale correspondant à la musique préférée du conducteur devrait le placer à un niveau d'activation optimal en augmentant son niveau d'activation.

Afin de quantifier en termes de performances de conduite les modulations du niveau d'activation induites par la musique, nous avons choisi une tâche de suivi de véhicule. Cette tâche spécifique a été choisie car elle nécessite l'attention soutenue du conducteur qui doit ajuster en permanence la vitesse de son véhicule à celle du véhicule à suivre $[12,13]$. La notion d'attention soutenue sous-tendue par cette tâche de suivi est entendue au sens du maintien d'un « niveau d'efficience adéquat et stable au cours d'une activité d'une certaine durée sollicitant un contrôle attentionnel continu » [34]. La tâche de suivi de véhicule implique un processus continu d'ajustement de la vitesse basé sur l'analyse des déplacements du véhicule suivi, ce qui a pour effet de mobiliser durablement les ressources attentionnelles du conducteur.

La piste musicale préférée des conducteurs a donc été choisie pour les placer à un niveau d'activation optimal avant modification du tempo. Ceci permettra de tester l'hypothèse d'une sur-activation avec une augmentation du tempo musical et d'une sous-activation avec une diminution du tempo musical en référence au niveau d'activation générée par le morceau musical préféré actuel des conducteurs. Les conducteurs étaient confrontés à quatre conditions associées à des environnements sonores différents 1. sans musique, une condition contrôle, 2 avec leur musique préférée du moment, supposée induire une activation optimale, 3 . avec leur musique légèrement accélérée, supposée induire une sur-activation et 4 . avec leur musique légèrement décélérée supposée induire une sous-activation. Une amélioration des performances de conduite était attendue en présence de la musique préférée des conducteurs comparativement à la condition sans musique. La modification du tempo devait quant à elle conduire à une détérioration des performances par sous- ou sur-activation selon que le tempo ait été ralenti ou accéléré respectivement.

\section{Méthode}

\section{Participants}

L'échantillon expérimental était constitué de vingt-quatre individus recrutés grâce à des listes de diffusion ou par voie d'affichage notamment sur le campus de l'Université Lyon 2. Les participants étaient âgés de 18 à 40 ans (22 ans en moyenne $\pm 4,4$ ans) et disposaient d'une expérience de conduite comprise entre 1 et 22 ans (4,2 ans en moyenne \pm 4,1 ans). Les participants ont déclaré parcourir entre 1000 et 27000 kilomètres par an (9 $075 \mathrm{~km}$ en moyenne \pm 8566$)$. Tous ont pris part de manière volontaire à l'expérience sans aucune compensation et l'expérience a reçu une autorisation du comité d'éthique local. Aucun participant n'a souffert du mal du simulateur.

\section{Simulation et tâche de conduite}

Un mini-simulateur de conduite composé d'un ordinateur de type PC (Dell Optiplex 380 MT, processeur Intel Core Duo 2 E7500, 4 Go de RAM), d'un volant et d'un pédalier à retour de force ainsi que d'une boîte de vitesses a été utilisé (Logitech G27®)). L'environnement virtuel était présenté sur un écran d'une diagonale de 19 pouces situé à $75 \mathrm{~cm}$ des yeux des participants (soit un champ visuel d'environ $31^{\circ}$ horizontalement et $20^{\circ}$ verticalement). Le logiciel libre OpenSD2S a été utilisé pour générer l'environnement simulé (Institut Image - Le2i/CNRS, ENSAM \& Renault).

Un même scénario de conduite a été utilisé pour les différentes conditions expérimentales de l'étude. Il comprenait une double voie à sens de circulation opposés, sans aucun trafic, et se décomposait en deux phases : 1 . une phase de suivi d'un véhicule et 2 . une phase de conduite libre. Lors de la phase de suivi, il était demandé aux participants de suivre un véhicule qui démarrait une fois qu'ils avaient enclenché le contact de leur propre véhicule à une distance d'environ 40 mètres. Cette phase de suivi prenait place sur une distance de $3 \mathrm{~km}$ et pour une durée d'environ 3 minutes. Le véhicule suivi, après une phase d'accélération initiale, évoluait selon une vitesse variable, comprise entre 30 et $70 \mathrm{~km} / \mathrm{h}$, avec un facteur d'accélération constant $\left(\mathrm{a}=2,2 \mathrm{~m} \cdot \mathrm{s}^{-2}\right)$. L'amplitude des variations de vitesse du véhicule suivi impliquait des ajustements de vitesse fréquents et assez importants. La phase de conduite libre débutait immédiatement après que le véhicule suivi s'était garé sur le bord de la chaussée. Les participants avaient alors pour consigne de conduire comme ils le feraient s'ils étaient au volant de leur véhicule. Cette phase de conduite libre durait environ une minute. Pour les deux phases de conduite il était demandé aux participants de 
maintenir leur véhicule dans leur voie de circulation (à droite de la chaussée).

\section{Morceaux musicaux utilisés}

Avant la participation à l'expérience proprement dite, il était demandé aux participants de sélectionner et d'apporter, dans un format numérique, leur morceau musical préféré du moment. La piste musicale propre à chaque participant était utilisée pour l'ensemble des conditions musicales de l'étude. Ainsi chaque participant était confronté à une situation de conduite avec écoute de sa piste musicale préférée sans aucune modification, avec une accélération du tempo de $10 \%$ du tempo musical initial et avec une décélération de $10 \%$ du tempo musical initial. À ces trois conditions de conduite avec fond musical s'ajoutait une condition contrôle sans musique. L'ordre de présentation des quatre conditions expérimentales était contrebalancé en suivant un carré latin.

Le logiciel MixMeister BPM Detector a permis de mesurer le tempo musical des différents morceaux utilisés dans le cadre de l'étude. La modification seule du tempo musical sans modification des autres paramètres des morceaux musicaux, notamment la hauteur, a été réalisée via le logiciel Audacity® (http://audacity.sourceforge.net) et la librairie SoundTouch associée. Toutes les conditions musicales étaient diffusées à un volume sonore de $75 \mathrm{~dB}$.

\section{Procédure}

Lors du premier contact qui servait à établir le jour et l'heure de l'expérimentation, il était également demandé aux participants de sélectionner leur musique préférée du moment et de l'apporter le jour de l'expérimentation.

Le jour de l'étude, les participants remplissaient un formulaire de consentement. Ils étaient ensuite équipés de trois électrodes reliées à un dispositif d'amplification et d'enregistrement du signal cardiaque (Biopac $($ )). L'expérimentateur invitait ensuite les participants à s'installer dans le siège du simulateur de conduite et à réaliser une première fois les tâches de suivi et de conduite libre afin de se familiariser avec l'environnement de conduite.

Une fois équipé du dispositif d'enregistrement de la fréquence cardiaque et familier de l'environnement de conduite, l'étude en elle-même débutait. Chaque conducteur était alors confronté aux quatre conditions expérimentales de l'étude dans un ordre contrebalancé 1. SM (Sans Musique), 2. M (Musique jouée au tempo normal), 3. M+10 (Musique au tempo accéléré de $10 \%$ ), 4. M-10 (Musique au tempo décéléré de $10 \%$ ).

Afin d'évaluer l'humeur des participants en fonction des différents fonds musicaux, ils leur était demandé de compléter le questionnaire Brief Mood Introspection Scale (BMIS) [35] après la réalisation de chaque condition expérimentale.

\section{Analyse des données}

Des données de trois natures différentes ont été analysées. Premièrement, nous avons analysé des données de nature physiologique, et calculé la fréquence cardiaque moyenne et son écart-type par condition expérimentale à l'aide du logiciel AcqKnowledge $囚$ (Biopac Systems, Inc.). Ces indicateurs physiologiques ont été utilisés pour évaluer le niveau d'activation des conducteurs [13, 22, 36]. Deuxièmement l'état subjectif émotionnel a été évalué, après chaque condition expérimentale, à l'aide du questionnaire BMIS. Enfin nous avons analysé les données relatives à la conduite en elle-même. Pour cela, plusieurs indicateurs ont été calculés lors de la phase de suivi du véhicule : a) le Temps inter-véhiculaire (TIV) temps séparant le véhicule suivi du véhicule conduit, calculé à une fréquence de $60 \mathrm{~Hz}$ et moyenné sur l'ensemble de la phase de suivi et trois indicateurs spécifiques à l'analyse d'une tâche de suivi de véhicule [37], b) la cohérence, ratio compris entre 0 et 1 qui rend compte du lien entre les changements de vitesse entre le véhicule piloté et le véhicule suivi, c) le délai, représentant le temps mis par le conducteur pour réagir aux changements de vitesse du véhicule suivi, d) et le gain, un facteur d'amplification qui qualifie l'amplitude de la réaction du conducteur lors des changements de vitesse du véhicule suivi. Concernant la phase de conduite libre les moyennes des vitesses de conduite libre ont été analysées.

Des ANOVAs à mesures répétées ont été utilisées pour l'ensemble des analyses statistiques. Un seuil de significativité de $p<0,05$ a été adopté pour toutes les inférences statistiques, les valeurs de $p$ comprises entre 0,05 et 0,1 ont été considérées comme des tendancielles. Les analyses post-hoc ont été calculées à l'aide du test LSD de Fisher.

\section{Résultats}

\section{Activité cardiaque}

La fréquence cardiaque moyenne et la variabilité de la fréquence cardiaque dans chaque environnement sonore ont été retenues comme indicateurs physiologiques du niveau d'activation des participants. Compte tenu de problèmes techniques rencontrés pour certains enregistrements, les données relatives à l'activité cardiaque de cinq participants n'ont pas pu être exploitées. Par conséquent cinq participants n'ont pas été inclus dans les analyses de l'activité cardiaque. 


\section{Fréquence cardiaque}

La fréquence cardiaque moyenne était significativement modifiée en fonction de l'environnement sonore auquel les participants étaient confrontés $(\mathrm{F}(3,51)=4,3, p<0,01$; voir Figure 1). Les tests post-hoc indiquent que la fréquence cardiaque moyenne enregistrée dans la condition Sans Musique (SM) diffère significativement de celle enregistrée dans les conditions musicales avec modification du tempo musical $(\mathrm{M}-10$ et $\mathrm{M}+10, p<0,05)$.

Une différence tendancielle est également enregistrée sans modification du tempo de la musique ( $\mathrm{M}, p<0,1)$. Concernant la comparaison des trois conditions musicales entre elles $(\mathrm{M}, \mathrm{M}+10, \mathrm{M}-10)$, seule une différence tendancielle entre $\mathrm{M}$ et $\mathrm{M}+10$ est apparue $(p<0,1)$. Une augmentation de la fréquence cardiaque est associée à une augmentation du tempo d'un même morceau musical.

\section{Variabilité de la fréquence cardiaque}

La variabilité de la fréquence cardiaque moyenne n'a pas été affectée significativement par l'environnement sonore $(\mathrm{F}(3$, 51) $=0,9$, NS ; voir Figure 2).

\section{État émotionnel subjectif}

Deux dimensions de l'état émotionnel des participants ont été évaluées via le questionnaire Brief Mood Introspection Scale (BMIS) : le niveau d'activation des participants (Figure 3 ) et le caractère plaisant de la situation (Figure 4).

\section{Niveau d'activation}

L'environnement sonore a significativement modifié le niveau d'activation déclaré par les participants $(\mathrm{F}(3$, $69)=7,7, p<0,001)$. Les analyses post-hoc ont montré que le niveau d'activation en environnement sonore SM diffère significativement de chacun des autres niveaux, dans les autres environnements sonores $(p<0,05)$.

Concernant les conditions musicales entre elles, la condition $\mathrm{M}+10$ tend à générer un niveau d'activation plus important que les conditions $\mathrm{M}$ et $\mathrm{M}-10(p<0,1)$.

\section{Caractère plaisant de la situation}

L'environnement sonore a modifié significativement le caractère plaisant de la situation $(\mathrm{F}(3,69)=2,8, p<0,05)$. Les analyses post-hoc indiquent que la condition SM se distingue significativement des conditions $\mathrm{M}$ et $\mathrm{M}+10$ ( $p$ $<0,05)$. La condition SM est jugée moins plaisante que les conditions $\mathrm{M}$ et $\mathrm{M}+10$.
Aucune différence n'est apparue entre les trois conditions musicales (M, M+10, M-10).

\section{Performances de conduite}

\section{Performances de conduite lors du suivi de véhicule}

Temps inter-véhiculaire (TIV). Le temps laissé par le conducteur entre son véhicule et le véhicule suivi, et qui traduit la marge de sécurité que se donne le conducteur, n'a pas été modifié par l'environnement sonore $(\mathrm{F}(3,69)=1,9$, NS ; Figure 5).

Cohérence, délai et gain. Pour compléter les résultats obtenus avec le TIV, une analyse plus fine des performances de la tâche de suivi a été réalisée. Il ressort de cette analyse une absence de différence significative entre les différents environnements sonores en matière de performances de suivi (voir Table 1). La cohérence entre les vitesses des deux véhicules $(\mathrm{F}(3,69)=0,73$; NS), le délai pris par les conducteurs pour répondre aux changements de vitesse du véhicule suivi $(\mathrm{F}(3,69)=0,48 ; \mathrm{NS})$ et le gain lié à ces changements de vitesse $(\mathrm{F}(3,69)=1,26$; NS) n'ont pas été significativement affectés par l'environnement sonore.

Vitesses de conduite libre. L'analyse statistique des moyennes des vitesses de conduite libre révèle des vitesses très proches les unes des autres entre les différents environnements sonores $(\mathrm{F}(3,69)=0,6$, NS avec SM : $57,9 \mathrm{~km} / \mathrm{h}, \mathrm{M}: 58 \mathrm{~km} / \mathrm{h}, \mathrm{M}+10: 58,6 \mathrm{~km} / \mathrm{h}, \mathrm{M}-10$ : $58,9 \mathrm{~km} / \mathrm{h})$.

\section{Discussion}

Les résultats obtenus en termes de fréquences cardiaques moyennes et de niveau d'activation subjectif (questionnaire BMIS) concordent et indiquent que la présence d'une musique en fond sonore a bien modifié le niveau d'activation des participants à l'étude. Les fréquences cardiaques moyennes (malgré l'impossibilité d'exploiter les données cardiaques pour 5 participants) et le niveau d'activation subjectif recueillis en condition sans musique se distinguent de manière quasi-systématique des trois conditions avec un fond sonore. Il apparaît donc clairement que le niveau d'activation est modifié en présence de musique. Toutefois, les légers changements du tempo de la musique (plus ou moins $10 \%$ de variation par rapport à la piste musicale originale) ont modifié le niveau d'activation des participants de manière tendancielle uniquement. Cette tendance est observée entre la musique sans modification de tempo en 


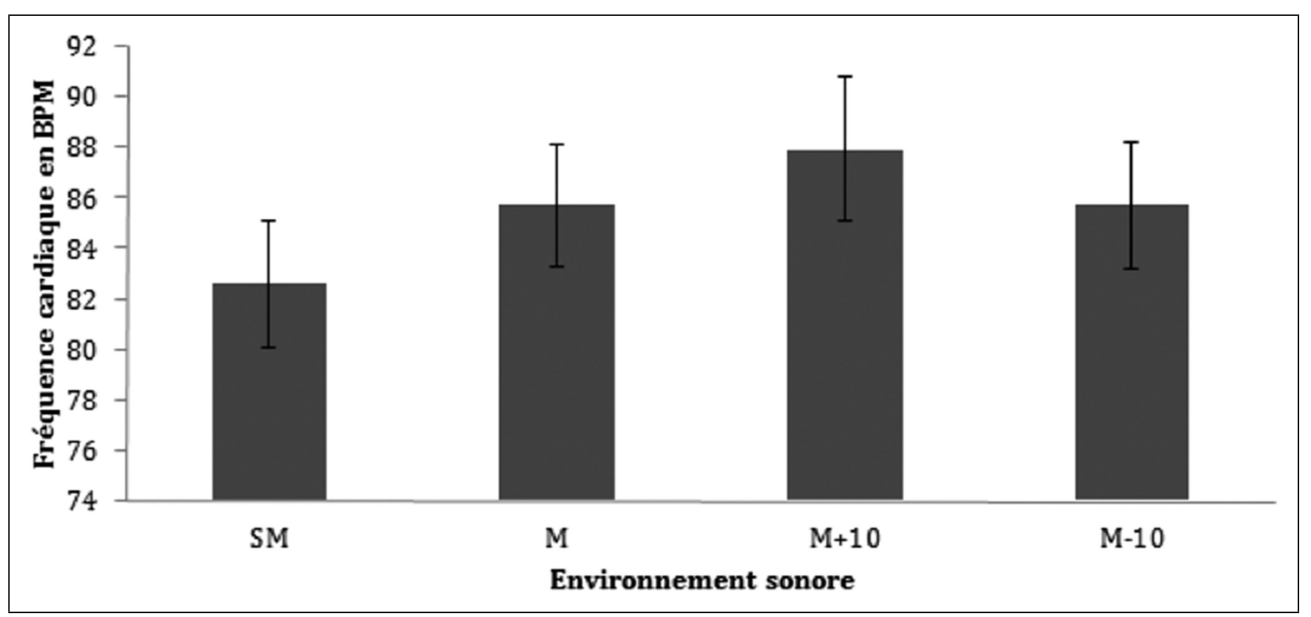

Fig. 1 Fréquence cardiaque moyenne exprimée en battements par minute (BPM) dans les quatre environnements sonores. Les barres d'erreur représentent l'erreur-type.

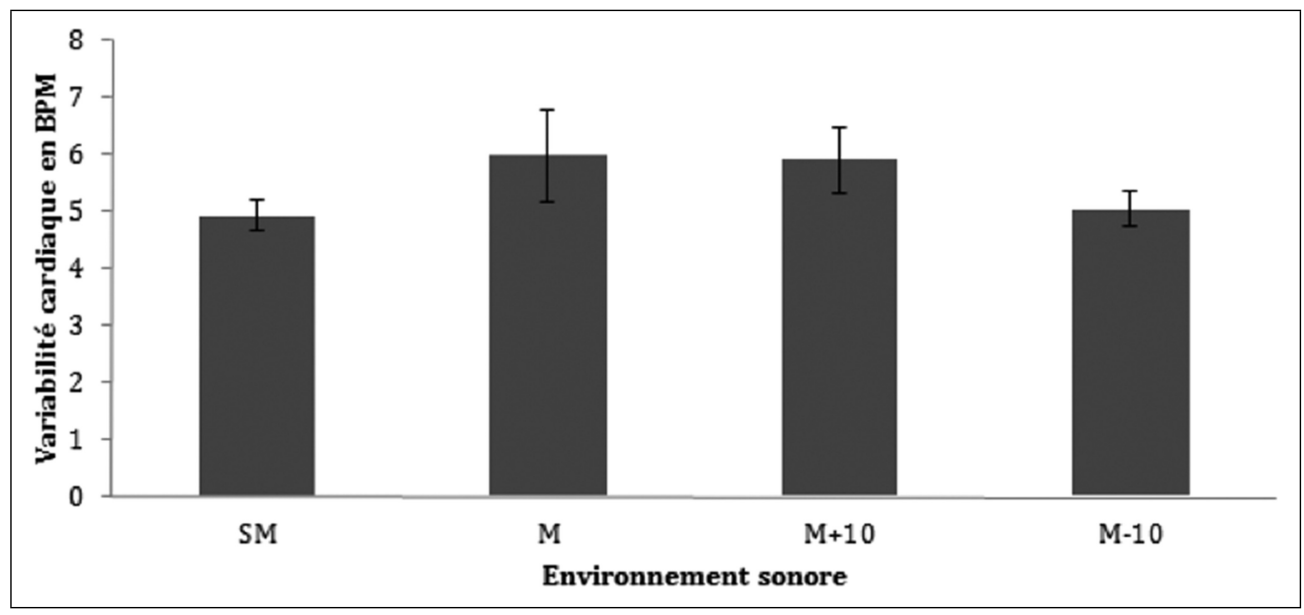

Fig. 2 Variabilité de la fréquence cardiaque moyenne exprimée en battements par minute (BPM) dans les quatre environnements sonores. Les barres d'erreur représentent l'erreur-type.

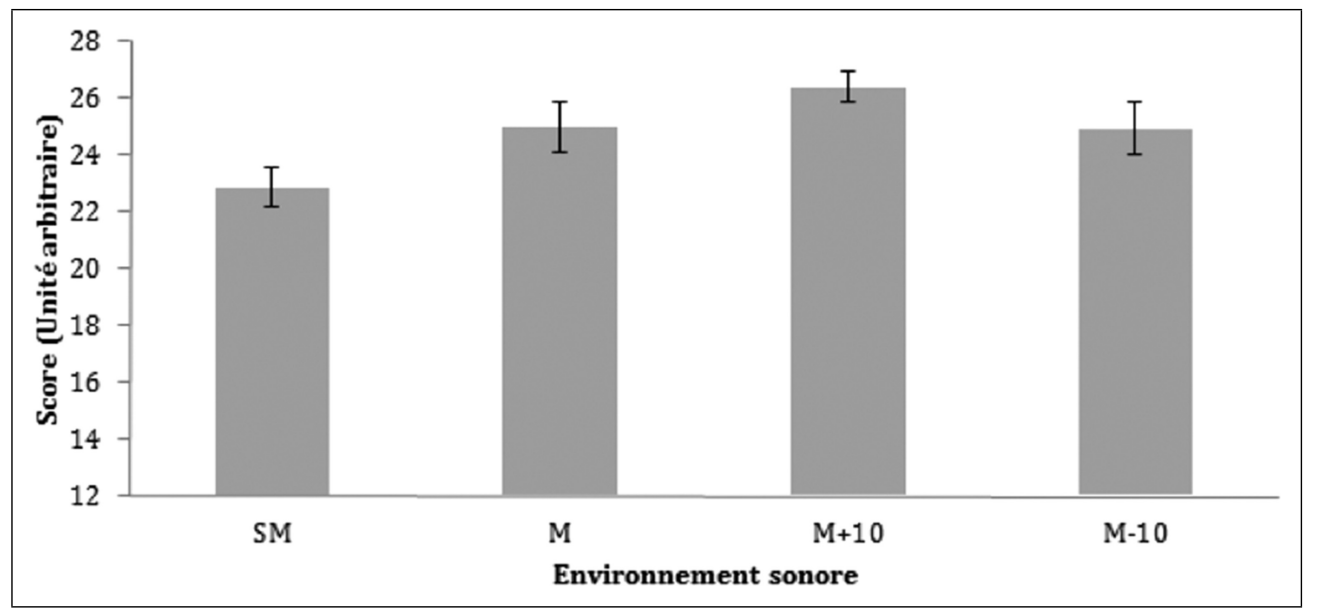

Fig. 3 Niveau d'activation dans les quatre environnements sonores. Échelle de mesure comprise entre 12 (niveau activation minimal) à 48 (niveau d'activation maximale). Les barres d'erreur représentent l'erreur-type. 


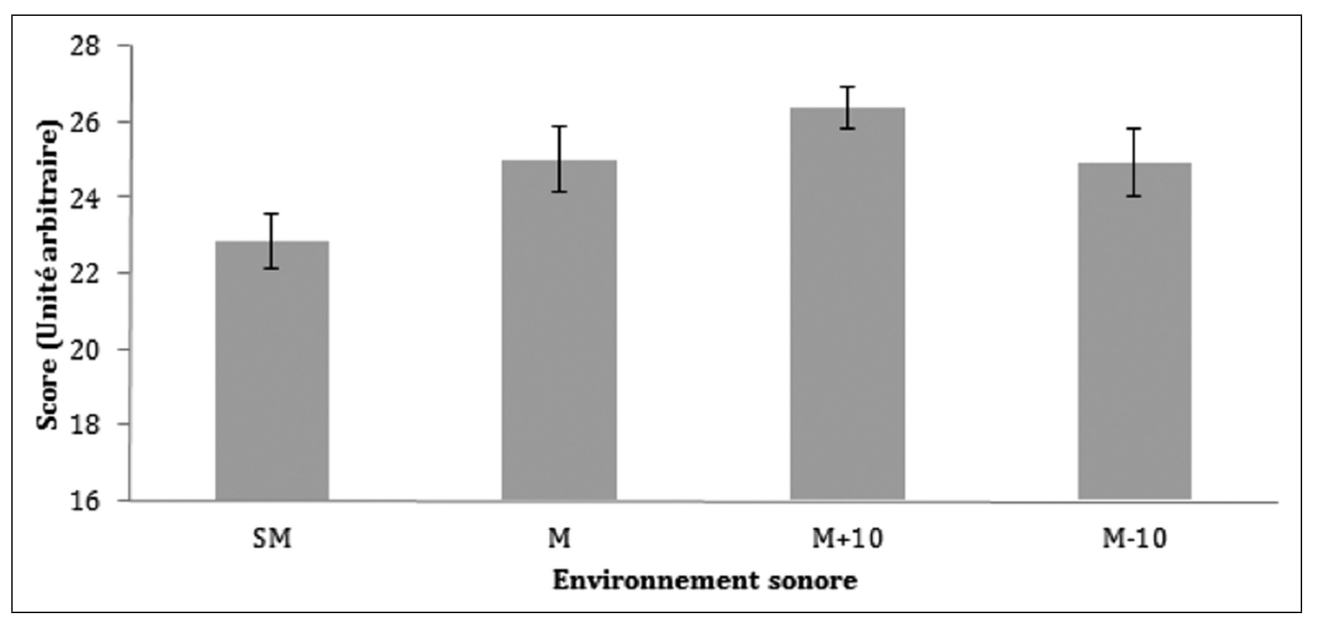

Fig. 4 Caractère plaisant de la situation dans les quatre environnements sonores. Échelle de mesure comprise entre 16 (situation la moins plaisante possible) à 64 (situation la plus plaisante possible). Les barres d'erreur représentent l'erreur type.

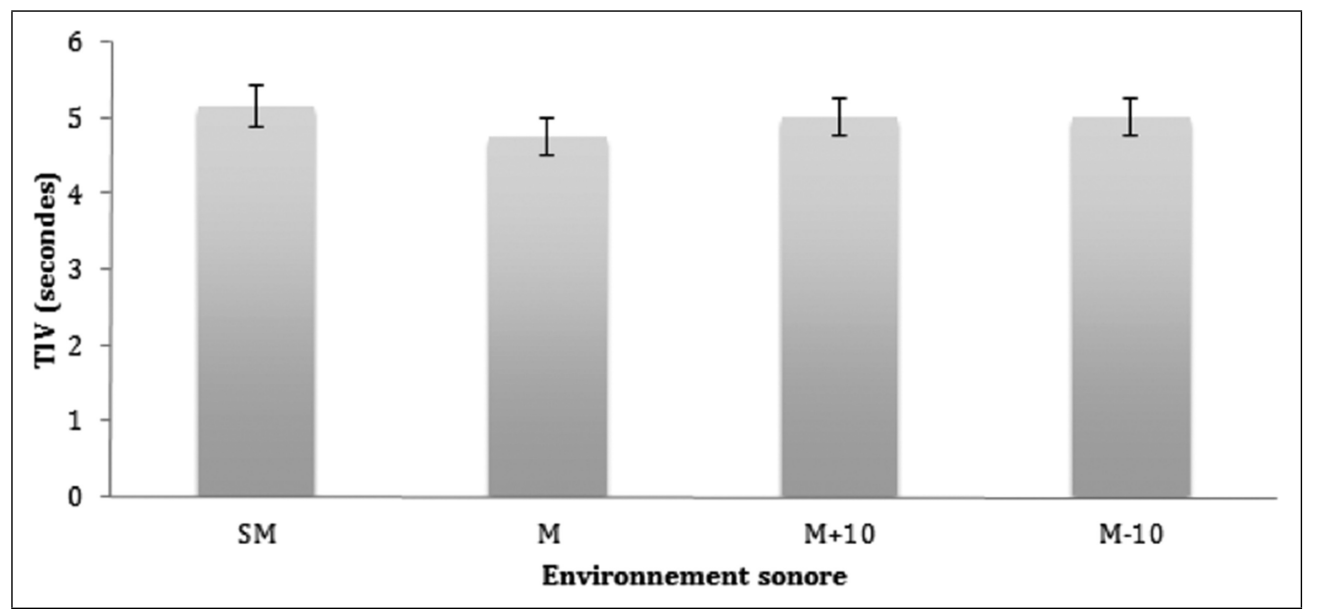

Fig. 5 Temps inter-véhiculaire moyen pour les différents environnements sonores. Les barres d'erreur représentent l'erreur-type.

Tableau 1 Synthèse des résultats obtenus pour la cohérence, le délai et le gain pour les quatre environnements sonores (moyenne \pm écart-type).

\begin{tabular}{|c|c|c|c|c|}
\hline & SM & $\mathbf{M}$ & $\mathrm{M}+10$ & M-10 \\
\hline Cohérence (ratio $0<\mathrm{x}<1$ ) & $0,83 \pm 0,11$ & $0,86 \pm 0,1$ & $0,83 \pm 0,11$ & $0,81 \pm 0,15$ \\
\hline Délai (s) & $4,74 \pm 5,89$ & $3,26 \pm 4,15$ & $3,81 \pm 4,56$ & $3,42 \pm 3,1$ \\
\hline Gain & $0,87 \pm 0,16$ & $0,90 \pm 0,13$ & $0,88 \pm 0,14$ & $0,86 \pm 0,17$ \\
\hline
\end{tabular}

comparaison à la musique accélérée de $10 \%$, à la fois pour l'évaluation subjective et physiologique du niveau d'activation (BMIS et fréquences cardiaques). Néanmoins, seule l'évaluation subjective tend à indiquer que la musique accélérée de $10 \%$ augmente le niveau d'activation en comparaison à la condition musicale ralentie de $10 \%$.
Ces changements semblent confirmer le fait que le tempo musical est à même de modifier le niveau d'activation des conducteurs. En revanche les modifications du niveau d'activation n'ont pas engendré de modification des performances de conduite. Ni les performances de suivi du véhicule ni les vitesses de conduite spontanées dans la 
partie de conduite libre ne sont modifiées par les différents environnements musicaux évalués.

S'il est indéniable que la musique est à même d'influencer le niveau d'activation et l'état émotionnel, quels que soient le pays et la culture considérée, tous les individus ne sont pas sensibles de la même manière à la musique. Autrement dit, un même morceau musical peut avoir des répercussions différentes sur des individus différents. Il a d'ailleurs été mis en évidence que les goûts musicaux et plusieurs dimensions de la personnalité sont corrélés [3]. En conséquence chaque individu doit pouvoir choisir sa musique préférée afin que l'environnement sonore soit positif et le place dans des conditions d'activation optimum. C'est le choix qui a été fait dans cette étude où les participants ont apporté leur morceau de musique préféré du moment. Les questionnaires BMIS ont indiqué qu'en présence de leur musique préférée les conducteurs estimaient la situation plus plaisante qu'en l'absence de musique. Ces résultats confirment des observations précédentes selon lesquelles, lorsque leur musique préférée est jouée en arrière-plan, les conducteurs voient leur humeur s'améliorer et prennent plus de plaisir à la conduite [17]. Les modifications de tempo de leur piste musicale préférée n'ont pas affecté significativement le caractère plaisant de la situation. Par conséquent il semble qu'avoir offert la possibilité aux participants de choisir leur propre piste musicale ait été un choix judicieux car leur humeur était affectée de manière similaire pour les trois conditions musicales (piste originale, piste accélérée de $10 \%$ et piste décélérée de $10 \%$ ). L'induction émotionnelle opérée par la musique $[18,20]$ a ainsi été maintenue constante. Il s'agit d'un résultat d'importance indiquant que l'état émotionnel généré par la musique aux différents tempos est similaire et ne vient pas interférer avec la manipulation du tempo.

Le fait d'avoir laissé le choix aux participants de leur piste musicale préférée avait également pour objectif de placer les conducteurs dans une fenêtre d'activation optimale. Les résultats indiquent une augmentation du niveau d'activation en présence de la musique sans modification de tempo. Ceci laisse à penser que l'écoute de sa musique préférée du moment est un activateur pour le participant. Une accélération du tempo de cette même musique entraîne une activation physiologique et subjective encore plus grande que celle observée pendant l'écoute de la musique dans sa version originale. Une accélération du tempo de la musique est donc en mesure de modifier le niveau d'activation de manière tendancielle. En revanche nos résultats n'indiquent pas une activation moindre lors de la décélération du tempo musical. Par ailleurs les données de conduite n'indiquent pas de différences entre les quatre environnements sonores associés à la conduite. Compte tenu de ces résultats qui ne correspondent que partiellement aux hypothèses, il n'est pas possible d'affirmer que la fenêtre d'activation optimale est atteinte en faisant un usage direct de la musique préférée des participants.

La contrepartie de cette liberté de choix des morceaux musicaux est la variabilité des stimuli musicaux utilisés. Les pistes musicales choisies avaient des tempos allant de 76 à 172 battements par minute (115 BPM en moyenne \pm 23) dans leur version originale. Compte tenu du fait que le tempo original était dépendant de morceau de musique, les modifications de tempo (plus ou moins $10 \%$ ) pourraient ne pas avoir eu exactement les mêmes effets sur le niveau d'activation et la conduite. Pour l'environnement sonore M+10, les tempos étaient compris entre 83 à 190 BPM (127 BPM en moyenne \pm 26 ) tandis que pour l'environnement sonore $\mathrm{M}-10$ les tempos étaient compris entre 68 et 155 BPM (104 BPM en moyenne \pm 21$)$. À titre de comparaison l'étude de Brodsky citée en introduction avait utilisé des pistes musicales comprises entre 40 et 70 BPM pour des musiques dites lentes, entre 85 et 110 BPM pour des musiques dites intermédiaires et entre 120 et $140 \mathrm{BPM}$ pour des musiques dites rapides [22]. Dans la présente étude, les tempos tous participants confondus pour les conditions M-10 (104 BPM), MN (115,5 BPM) et M+10 (127 BPM) sont compris dans un intervalle allant de 104 à 127 BPM, soit une plage de tempos bien moins large que celle allant de 40 à 140 BPM utilisée par Brodsky.

Brodsky n'avait pas observé de changement significatif de fréquence cardiaque dans les différentes conditions musicales rapportées dans son étude, contrairement aux observations rapportées ici [22]. Il rapportait en revanche une augmentation des vitesses de conduites spontanées, du nombre de franchissements de bords de voie et du nombre de non-respect de feu rouge de plus en plus importante avec l'augmentation du tempo des musiques utilisées. Une augmentation quasi-linéaire des vitesses de conduite spontanées en fonction du tempo musical joué en arrière-plan était rapportée. Les pistes musicales avec un tempo lent étaient associées avec une vitesse moyenne de $132 \mathrm{~km} / \mathrm{h}$, le tempo intermédiaire avec une vitesse moyenne de $136 \mathrm{~km} / \mathrm{h}$ et le tempo le plus rapide avec une vitesse moyenne $140 \mathrm{~km} / \mathrm{h}$. Il est important de noter toutefois que ces vitesses de conduites spontanées moyennes obtenues sur un simulateur sont plus de deux fois supérieures à celles enregistrées dans la présente étude (légèrement en dessous de $60 \mathrm{~km} / \mathrm{h}$ ). Les vitesses rapportées par Brodsky ne sont pas réalistes considérant l'environnement urbain et périurbain dans lequel ont évolué les participants. Il s'agissait de la simulation du périphérique de la ville de Chicago avec des portions de route municipales limitées à $50 \mathrm{~km} / \mathrm{h}$ et des portions de route inter-états limitées à $105 \mathrm{~km} / \mathrm{h}$. Les vitesses de conduites spontanées obtenues par Brodsky indiquent que les conducteurs n'étaient pas 
dans des conditions de conduites très réalistes et que les variations obtenues entre conditions musicales sont à considérer avec précaution. Dans la présente étude, le choix de se focaliser sur l'impact de la musique sur l'activité de guidage du véhicule a été fait, ceci car il s'agit d'une sous-tâche de conduite en lien direct avec les boucles de contrôle perceptivo-motrices ou habiletés du conducteur [38]. Aucun des indicateurs utiles à l'analyse de la tâche de suivi (temps inter-véhiculaire, cohérence, délai, gain) ou les vitesses spontanées pour la partie de l'expérience en conduite libre n'ont été affectés par les différents environnements sonores. Le fait que les modifications de tempo à travers nos conditions expérimentales soient finalement trop faibles pourrait être une explication à cela. Il est toutefois surprenant de ne pas avoir observé d'effet de la musique sans modifications sur la tâche de guidage du véhicule. Se pose alors la question de la sensibilité de la tâche de suivi aux effets de la musique. Il était attendu de cette tâche qu'elle mobilise l'attention des conducteurs de manière continue et soit donc particulièrement sensible aux changements d'activation. Des études antérieures valident le choix de cette tâche de suivi comme une tâche adaptée aux objectifs poursuivis dans cette étude [13, 39].

Les résultats obtenus invitent toutefois à questionner le choix de cette tâche comme étant la meilleure option afin de mettre en évidence les effets de la musique sur la conduite. Peut-être que les autres grandes tâches de conduite que sont l'identification de risques (ex. piétons, rétrécissement de chaussée, obstacle sur la route...) et la navigation (ex. interactions avec un système de guidage embarqué, recherche d'un lieu inconnu) seraient-elles plus sensibles aux effets de la musique et de son tempo ? Il est également probable que les effets de la musique sur les performances de conduite ne soient pas très marqués en condition de vigilance «normale » telles qu'elles ont été imaginées pour cette étude. En effet, la musique à différents tempos devrait avoir des effets plus marqués avec des conducteurs en situations d'hypo- ou d'hyper-vigilance. Cette idée corroborerait bien les résultats décrits par Brodsky [22] dans la mesure où l'on considère que dans son étude, les conducteurs étaient très activés comme en témoignent les vitesses de conduites spontanées très élevées. En situation de forte activation (comme pour la faible activation d'ailleurs) les performances varient plus rapidement que dans la zone d'activation intermédiaire. Une piste de recherche pour la suite serait de placer les conducteurs dans un niveau d'activation qui ne soit pas intermédiaire au moment de les faire conduire avec de la musique.

Sur la base des données collectées lors de notre expérience, il n'est pas possible de conclure que le tempo musical (d'un même morceau) a une influence sur les performances de conduite. Cette absence d'effet est potentiellement attribuable à de trop petites modifications du tempo original de la musique. Le tempo de la musique est à même de modifier le niveau d'activation des conducteurs : plus le tempo est rapide et plus le niveau d'activation est élevé. Les différences observées dans cette étude sont cependant de petite taille et n'ont donné lieu qu'à des changements statistiquement tendanciels. Il est donc possible que l'environnement sonore n'ait pas modifié les performances de conduite en raison de ces trop petits effets sur le niveau d'activation, l'amplitude des modifications du tempo ayant été calibrée de manière à ne pas modifier de manière trop importante le morceau musical original. Il apparaît difficile de modifier plus le tempo musical d'une même piste sans en altérer le reste de sa musicalité. D'une certaine manière, une tentative allant dans le sens d'une modification plus importante du tempo d'une piste musicale reviendrait au même que de choisir des pistes musicales différentes. En effet la seule modification du tempo contaminerait alors les autres composantes émotionnelles de la musique, qui seraient affectées par cette modification. Une autre explication à l'absence de changement dans les performances de conduite en fonction du tempo musical serait que l'activité de guidage n'est pas affectée par le tempo musical. Ceci irait dans le sens des études qui n'indiquent pas d'effet de la musique de manière générale sur les performances de conduite [14, 15].

\section{Conclusion}

Une légère modification $( \pm 10 \%)$ du tempo de la musique préférée des participants a généré une légère modification du niveau d'activation rapporté chez ces mêmes participants. Toutefois les différents niveaux d'activation obtenus en fonction de l'environnement sonore ne transparaissent pas dans les performances de conduite enregistrées. D'autres études sont nécessaires avant de pouvoir conclure de manière ferme aux effets du tempo de la musique sur les performances de conduite. De plus, le niveau d'activation de base des conducteurs, le contexte de conduite, le rythme musical et les émotions générées par la musique sont autant de facteurs qui se combinent et sont à même d'influencer les comportements de conduite.

\section{Remerciements}

Cette étude a été réalisée dans le cadre du financement Labex Cortex (ANR-11-LABX-0042) de 1'Université de Lyon. Les auteurs remercient particulièrement les développeurs du logiciel OpenSD2S pour le développement de la tâche de suivi et leur support. Les auteurs sont également reconnaissants envers Manon Perdrix et Geoffrey 
Seccia pour leur aide, en particulier pour les passations expérimentales.

\section{Références}

1. Glacken, CJ (1973) Traces on the Rhodian shore: Nature and culture in Western thought from ancient times to the end of the eighteenth century. Univ of California Press.

2. Dubois, N, Moch, A (2006) L'automobile et le sentiment d'être chez-soi. Rev Eur Psychol Appl Rev Appl Psychol 56(2): 95-107.

3. Rentfrow, PJ, Gosling, SD (2003) The do re mi's of everyday life: the structure and personality correlates of music preferences. $J$ Pers Soc Psychol 84(6): 1236.

4. Dibben, N, Williamson, VJ (2007) An exploratory survey of invehicle music listening. Psychol Music 35(4): 571-89.

5. Roballey, TC, McGreevy, C, Rongo, RR, Schwantes, ML, Steger, PJ, Wininger, MA, et al. (1985) The effect of music on eating behavior. Bull Psychon Soc 23(3): 221-2.

6. Caldwell, C, Hibbert, SA (2002) The influence of music tempo and musical preference on restaurant patrons' behavior. Psychol Mark 19(11): 895-917.

7. Milliman, RE (1982) Using background music to affect the behavior of supermarket shoppers. J Mark: 86-91.

8. North, AC, Hargreaves, DJ, McKendrick, J (1999) The influence of in-store music on wine selections. J Appl Psychol 84(2): 271.

9. Edworthy, J, Waring, H (2006) The effects of music tempo and loudness level on treadmill exercise. Ergonomics 49(15): 1597610 .

10. Koger, SM, Chapin, K, Brotons, M (1999) Is music therapy an effective intervention for dementia? A meta-analytic review of literature. J Music Ther 36(1): 2-15.

11. Cuddy, LL, Duffin, J (2005) Music, memory, and Alzheimer's disease: is music recognition spared in dementia, and how can it be assessed? Med Hypotheses 64(2): 229-35.

12. Ünal, AB, Steg, L, Epstude, K (2012) The influence of music on mental effort and driving performance. Accid Anal Prev 48: 271-8.

13. Ünal, AB, de Waard, D, Epstude, K, Steg, L (2013) Driving with music: Effects on arousal and performance. Transp Res Part F Traffic Psychol Behav 21: 52-65.

14. Hatfield, J, Chamberlain, T (2008) The effect of audio materials from a rear-seat audiovisual entertainment system or from radio on simulated driving. Transp Res part F traffic Psychol Behav 11(1): 52-60.

15. Bellinger, DB, Budde, BM, Machida, M, Richardson, GB, Berg, WP (2009) The effect of cellular telephone conversation and music listening on response time in braking. Transp Res Part F Traffic Psychol Behav 12(6): 441-51.

16. Dalton, B, Behm, D, Kibele, A (2007) Effects of sound types and volumes on simulated driving, vigilance tasks and heart rate. Occup Ergon 7: 153-68.

17. Brodsky, W, Slor, Z (2013) Background music as a risk factor for distraction among young-novice drivers. Accid Anal Prev 59: 382 93

18. Pêcher, C, Lemercier, C, Cellier, J (2009) Emotions drive attention: Effects on driver's behaviour. Saf Sci 47(9): 1254-9.
19. Jallais, C, Gabaude, C, Paire-Ficout, L (2014) When emotions disturb the localization of road elements: Effects of anger and sadness. Transp Res part F traffic Psychol Behav 23: 125-32.

20. Van der Zwaag, M, Janssen, JH, Nass, C, Westerink, J, Chowdhury, S, de Waard, D (2013) Using music to change mood while driving. Ergonomics 15: 4-14.

21. Konz, S, Mcdougal, D (1968) The effect of background music on the control activity of an automobile driver. Hum Factors 10(3): 233-43.

22. Brodsky, W (2002) The effects of music tempo on simulated driving performance and vehicular control. Transp Res part $F$ traffic Psychol Behav 4: 219-41.

23. Yerkes, RM, Dodson, JD (1908) The relation of strength of stimulus to rapidity of habit-formation. J Comp Neurol Psychol 18: 459-82.

24. Hevner, K (1937) The Affective Value of Pitch and Tempo in Music. Am J Psychol 6: 21-30.

25. Holbrook, MB, Anand, P (1990) Effects of Tempo and Situational Arousal on the Listener's Perceptual and Affective Responses to Music. Psychol Music 18(2): 150-62.

26. Dillman Carpentier, FR, Potter, RF (2007) Effects of Music on Physiological Arousal: Explorations into Tempo and Genre. Media Psychol 10(3): 339-63.

27. Husain, G, Thompson, WF, Schellenberg, EG (2002) Effects of Musical Tempo and Mode on Arousal, Mood, and Spatial Abilities. Music Percept 20(2): 151-71.

28. Kahneman, D (1973) Attention and effort. New Jersey, USA: Prentice Hall.

29. Young, MS, Brookhuis, KA, Wickens, CD, Hancock, PA (2014) State of science: mental workload in ergonomics. Ergonomics: 37 41.

30. Schäfer, T, Sedlmeier, P (2011) Does the body move the soul? The impact of arousal on music preference. Music Percept 29(1): 37 50.

31. Hirokawa, E (2004) Effects of music listening and relaxation instructions on arousal changes and the working memory task in older adults. J Music Ther 41(2): 107.

32. De Jong, MA, Van Mourik, KR, Schellekens, HMC (1973) A physiological approach to aesthetic preference. Psychother Psychosom 22(1): 46-51.

33. Gomez, P, Danuser, B (2004) Affective and physiological responses to environmental noises and music. Int J Psychophysiol 53(2): 91-103.

34. Zomeren, AH, Brouwer, WH (1994) Clinical neuropsychology of attention. Oxford University Press.

35. Mayer, JD, Gaschke, YN (1988) The experience and MetaExperience of Mood. J Pers Soc Psychol 55: 102-11.

36. Mehler, B, Reimer, B, Coughlin, J, Dusek, J (2009) Impact of incremental increases in cognitive workload on physiological arousal and performance in young adult drivers. Transp Res Rec $J$ Transp Res Board 2138: 6-12.

37. Brookhuis, K, de Waard, D, Mulder, B (1994) Measuring driving performance by car-following in traffic. Ergonomics 37(3): 427 34.

38. Rasmussen, J (1986) Information Processing and Human-Machine Interaction. An Approach to Cognitive Engineering. Elsevier. Amsterdam: North-Holland.

39. Brackstone, M, Mcdonald, M (2000) Car-following: a historical review. Transp Res Part F Traffic Psychol Behav 2: 181-96. 
